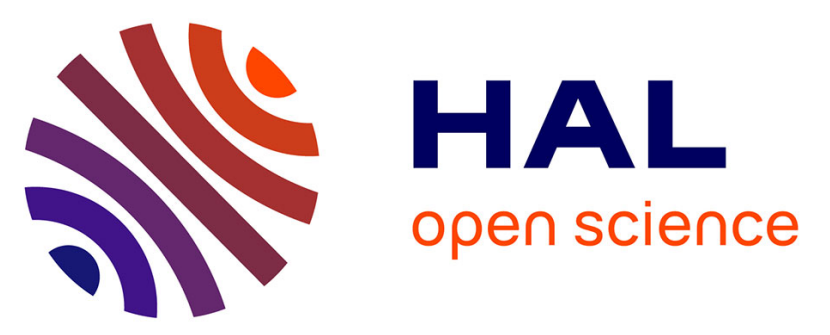

\title{
THE ADDITION OF CYCLOPHOSPHAMIDE TO LENALIDOMIDE AND DEXAMETHASONE IN MULTIPLY RELAPSED/RERACTORY MYELOMA PATIENTS; A PHASE I/II STUDY
}

Stephen Schey, Gareth J Morgan, Karthik Ramasamy, Beth Hazel, Sophie Corderoy, Dariusz Ladon, Mathew Jenner, Karen Phekoo, Kevin David Boyd, Faith E Davies

\section{- To cite this version:}

Stephen Schey, Gareth J Morgan, Karthik Ramasamy, Beth Hazel, Sophie Corderoy, et al.. THE ADDITION OF CYCLOPHOSPHAMIDE TO LENALIDOMIDE AND DEXAMETHASONE IN MULTIPLY RELAPSED/RERACTORY MYELOMA PATIENTS; A PHASE I/II STUDY. British Journal of Haematology, 2010, 150 (3), pp.326. 10.1111/j.1365-2141.2010.08250.x * hal-00552599

\author{
HAL Id: hal-00552599 \\ https://hal.science/hal-00552599
}

Submitted on 6 Jan 2011

HAL is a multi-disciplinary open access archive for the deposit and dissemination of scientific research documents, whether they are published or not. The documents may come from teaching and research institutions in France or abroad, or from public or private research centers.
L'archive ouverte pluridisciplinaire HAL, est destinée au dépôt et à la diffusion de documents scientifiques de niveau recherche, publiés ou non, émanant des établissements d'enseignement et de recherche français ou étrangers, des laboratoires publics ou privés. 


\section{b] british journal of haematology}

\section{THE ADDITION OF CYCLOPHOSPHAMIDE TO LENALIDOMIDE AND DEXAMETHASONE IN MULTIPLY RELAPSED/RERACTORY MYELOMA PATIENTS; A PHASE I/II STUDY}

\begin{tabular}{|c|c|}
\hline Journal: & British Journal of Haematology \\
\hline Manuscript ID: & BJH-2010-00300.R1 \\
\hline Manuscript Type: & Ordinary Papers \\
\hline $\begin{array}{r}\text { Date Submitted by the } \\
\text { Author: }\end{array}$ & 07-Apr-2010 \\
\hline Complete List of Authors: & $\begin{array}{l}\text { Schey, Stephen; Kings College Hospital Foundation NHS Trust and } \\
\text { Kings College London, Haematology } \\
\text { morgan, gareth; Institute of Cancer Research, Section of Haemato- } \\
\text { oncology } \\
\text { Ramasamy, Karthik; Kings College Hospital, Haematological } \\
\text { Medicine; Kings College London } \\
\text { Hazel, Beth; Kings College Hospital Foundation NHS Trust, } \\
\text { Haematology } \\
\text { Corderoy, Sophie; Kings College Hospital Foundation NHS Trust, } \\
\text { Haematology } \\
\text { Ladon, Dariusz; Kings College Hospital Foundation NHS Trust, } \\
\text { Haematology } \\
\text { Jenner, Mathew; Institute of Cancer Research, Section of Haemato- } \\
\text { oncology } \\
\text { Phekoo, Karen; Kings College Hospital Foundation NHS Trust, } \\
\text { Haematology } \\
\text { boyd, kevin; The Royal Marsden Hospital, Haemato-oncology } \\
\text { Davies, Faith; Institute of Cancer Research, Section of Haemato- } \\
\text { oncology }\end{array}$ \\
\hline Key Words: & MYELOMA, CORTICOSTEROIDS, THERAPY \\
\hline
\end{tabular}


THE ADDITION OF CYCLOPHOSPHAMIDE TO LENALIDOMIDE AND DEXAMETHASONE IN MULTIPLY RELAPSED/RERACTORY MYELOMA PATIENTS; A PHASE I/II STUDY

SA Schey ${ }^{1}$, GJ Morgan ${ }^{2}$, K Ramasamy ${ }^{1}$, B Hazel ${ }^{1}$, D Ladon ${ }^{1}$ S Corderoy ${ }^{1}$, M Jenner $^{2}, \mathrm{~K}$ Phekoo ${ }^{1}$, K Boyd ${ }^{2}$, FE Davies ${ }^{2}$

1. Kings College Hospital Foundation NHS Trust, London, UK

2. Royal Marsden NHS Foundation Trust, London, UK

Corresponding author:

Stephen A Schey

Department of Haematological Medicine

King's College Hospital

London

UK

Phone: $\quad+44203-299-4607$

Fax: $\quad+442032993514$

e-mail: sschey@nhs.net

Running Title: Lenalidomide with cyclophosphamide and dexamethasone in relapsed myeloma 


\section{Summary}

Two identical Phase III randomised trials confirmed the significant clinical activity of lenalidomide and dexamethasone in myeloma patients with relapsed disease. We report the results of a Phase $1 / I$ dose escalation study, which set out to evaluate the optimal dose of cyclophosphamide when used in combination with a fixed dose of Lenalidomide and dexamethasone in relapsed/refractory myeloma. A total of 31 patients were enrolled in cohorts of 3 patients at 5 dose levels of cyclophosphamide to a maximum dose of $700 \mathrm{mg}$ days 1 and 8 of a 28 day cycle. Patients received Lenalidomide $25 \mathrm{mg}$ days 1 through 21 and dexamethasone $20 \mathrm{mg}$ orally days $1-4$ and $8-11$. The maximum tolerated dose (MTD) of this regimen is $600 \mathrm{mg}$ cyclophosphamide days 1 and 8 and the regimen is well tolerated with grade $3 / 4$ haematological complications only occurring in $26 \%$ of patients, grade $3 / 4$ infection in $3 \%$ of patients, both at the $700 \mathrm{mg}$ cyclophosphamide dose level with thromboembolic complications in $6 \%$ of patients. The overall complete remission rate was $29 \%$, very good partial remission rate $7 \%$ and partial remission rate $45 \%$ giving an overall response rate of $81 \%$. After a median follow-up of 21 months, projected 2 year progression free survival (PFS) is $56 \%$ and the overall survival at 30 months is $80 \% .10$ patients were treated in an extended cohort group at the maximum tolerated dose of cyclophosphamide with a $40 \%$ CR rate. No dose reductions for any of the study drugs or deaths was required during cycles 1-9. The combination of Lenalidomide, cyclophosphamide and dexamethasone is a safe and effective combination in relapsed myeloma inducing a high response rate that are durable and warrants further investigation in the phase 3 setting. 


\section{Introduction:}

Immunomodulatory (IMiD) drugs are an important set of molecules in the management of myeloma patients. Thalidomide was first shown to be effective in relapsed/refractory myeloma(Singhal, et al 1999) with responses of partial response (PR) or greater being seen in $>20 \%$ of patients (YakoubAgha, et al 2002). Based on these and subsequent studies, thalidomide is currently used at all disease stages and often in combination with a steroid and alkylating agent. More recently, the role of Lenalidomide (Revlimid), a more potent IMiD, has been explored in patients with relapsed myeloma in two identical phase 3 studies (Dimopoulos, et al 2007, Weber, et al 2007). These studies compared Lenalidomide/Dexamethasone with Dexamethasone alone and demonstrated the combination improved progression free and overall survival.

Alkylating agents have been the standard of care for myeloma patients for many years with Melphalan and Prednisolone being considered the standard approach for patients not destined for autologous transplantation. A series of clinical trials performed in the UK in the 1980s, demonstrated the equivalent efficiency of cyclophosphamide to melphalan and highlighted its favourable toxicity profile with less profound myelosuppression(Augustson, et al 2005), lacking the stem cell toxicity of melphalan, making it safe to use in patients destined for transplant(Child, et al 2003). In addition, cyclophosphamide has a large non-renal component of elimination making it a safer drug for patients with renal impairment. 
Combination chemotherapy regimen exploiting synergistic affects of the individual components are often highly effective in oncology and there is a need to develop effective combinations for patients with myeloma.

The addition of an alkylating agent to standard chemotherapy (CVAMP), thalidomide (CTD or MTP) or bortezomib (MPV or CVD) has been shown to increase response rates and possibly to prolong survival(Berenson, et al 2006, Kropff, et al 2007, Palumbo, et al 2006, Raje, et al 1997, Sidra, et al 2006). However, the optimal dose of cyclophosphamide in combination with lenalidomide, which is known to cause myelosuppression, has not yet been defined. The aims of this study were to develop a safe alkylating agent, Lenalidomide, and steroid combination suitable for clinical use and evaluation in subsequent randomised clinical trials. To this end, the maximum tolerated dose and toxicity profile of cyclophosphamide when used in combination with Lenalidomide and dexamethasone for patients with relapsed refractory disease was determined. 


\section{METHODS}

This was an open label, dose escalation phase I/II study designed to determine the maximum tolerated dose (MTD) of the combination of cyclophosphamide, Lenalidomide and dexamethasone. Research was approved by the King's College Hospital institutional review board and Cambridgeshire ethics committee and all participants gave written informed consent. Cyclophosphamide was given to 5 cohorts of 3 patients each at increasing doses from $300 \mathrm{mg}$ po up to a maximum of $700 \mathrm{mg}$ cyclophosphamide on days 1 and 8 of a 28 day cycle in combination with dexamethasone $20 \mathrm{mg}$ po, daily on days $1-4$ and $8-11$; Lenalidomide was given at a dose of $25 \mathrm{mg}$ po, daily on days 1-21 in line with the MM009 and MM010 phase 3 clinical trial protocols. The scheduling of cyclophosphamide on day 1 and 8 was chosen to minimise the haematological toxicity that was observed when it was given in addition on days 15 and 22 in our previously reported study. A total of 9 induction courses were administered and patients were then maintained on Lenalidomide at the maximum tolerated dose until disease progression or unacceptable toxicity. A further 10 patients were treated at the MTD dose of cyclophosphamide to gain experience of the regimen at this dose level. All subjects received low dose aspirin (e.g. $75 \mathrm{mg}$ ) as thromboprophylaxis. Erythropoietin was not given routinely in this study nor was prophylactic antiviral or anti-fungal treatment. Growth factor support was allowed if the neutrophil count dropped below $1.0 \times 10^{9} / /$ prior to initiating the next course of treatment and was then given for each successive course. 
Patients diagnosed as relapsed myeloma, at first or subsequent relapse, were eligible for the study but were excluded if they had received chemotherapy, including corticosteroids, within 28 days of the start of treatment. Local radiotherapy for symptom relief for previously recognised myeloma deposits was allowed before starting treatment but not to new sites of disease if they developed during the study which was classified as disease progression. Patients were eligible if they had adequate renal function (serum creatinine $<2$ $\mathrm{mg} / / \mathrm{dl}$ ) and bone marrow reserve (baseline platelet count $>20 \times 10^{9} / \mathrm{l}$ or absolute neutrophil count $\left.>1.0 \times 10^{9} / \mathrm{l}\right)$. In addition, patients with grade 3 peripheral neuropathy or a history of other malignancy within 3 years were excluded.

\section{Dose limiting Toxicity}

Dose limiting toxicity (DLT) was defined as; Grade 4 haematological toxicity occurring during cycle 1 , febrile neutropenia during cycle 1 , grade $3 / 4$ nonhaematological toxicity during cycle 1 (excluding alopecia), or failure to start cycle 2 within 7 days of scheduled day due to treatment related toxicity. Providing no patient within a cohort experienced DLT, the subsequent cohort received an increased dose of cyclophosphamide. If one patient in a dose cohort experienced DLT, another three patients were enrolled at the same dose. If 2 or more of all 6 patients at that dose experienced DLT, the MTD would be determined as one cohort dose level below. If 1 or fewer of the 6 patients experienced DLT, three more patients were recruited into the subsequent, higher dose cohort, up to a maximum of $700 \mathrm{mg}$. . 
During the extended phase of the study, Lenalidomide was discontinued if the patient developed a desquamating rash or erythema multiforme any grade or sinus bradycardia or other cardiac arrhythmias $\geq$ grade 3 within a treatment cycle. Cyclophosphamide was withheld if patients developed cystitis grade $\leq 2$ and all therapy was withheld if other Grade $\geq 3$ non-haematological toxicity or grade 4 haematological toxicity occurred. If myelosuppression occurred following the second or subsequent course of treatment, Lenalidomide was discontinued until the neutrophil level was $>1.0 \times 10^{9} / \mathrm{l}$. A new course of treatment began on the scheduled day 1 of a new cycle if the ANC is $\geq$ $1,000 / \mu \mathrm{L}$, the platelet count is $\geq 50,000 / \mu \mathrm{L}$, and any lenalidomide-related allergic reaction/ hypersensitivity or sinus bradycardia/ other cardiac arrhythmia adverse event that may have occurred has resolved to $\leq$ grade 1 severity, or any other lenalidomide related adverse event that may have occurred had resolved to $\leq$ grade 2 severity. If these conditions were not met on day 1 of a new cycle, the subject was evaluated weekly and a new cycle of therapy initiated when the adverse event had resolved. If subjects could not initiate a new cycle of therapy within 28 days of the scheduled day 1 , the patient was withdrawn from therapy. The dose of Lenalidomide was reduced in subsequent courses by $5 \mathrm{mg}$ decrements and the patients' response assessed at weekly intervals whilst being continued on the initial dose of cyclophosphamide. Treatment was discontinued if there was evidence of disease progression or the patient developed unacceptable toxicity. Patients who discontinued treatment due to an adverse event were followed up to documented resolution or stabilisation of the event. In the event of a haematological DLT, patients were allowed to reduce the dose of Lenalidomide or cyclophosphamide and continue on treatment at the next 
reduced dose level, provided haematological recovery occurred within 4 weeks. Patients with stable disease were allowed to continue on study as long as clinical benefit was obtained. Subjects who discontinued treatment for any reason underwent a safety assessment 30 days post the last dose of study drug. At completion of cycle 9, subjects who had stable disease or better with an acceptable toxicity profile were able to continue on Lenalidomide as single agent maintenance therapy.

\section{Safety and Efficacy Assessment}

Patients were assessed weekly during the first month of treatment and 4 weekly thereafter. Response was assessed prior to each course of treatment, using the international uniform response criteria (Durie, et al 2006). Bone marrow aspirate/biopsy evaluations were carried out at trial entry and at discontinuation of treatment to confirm response. Adverse events were graded according to $\mathrm{NCl}$ common toxicity criteria (Version 3.0, Bethesda, MD, USA http:// ctep.cancer.gov/forms/ CTCv20_4-30-992.pdf). All patients who developed grade 4 haematological toxicity underwent bone marrow examination.

\section{Statistical Analysis:}

The primary outcome measures were toxicity and safety. The secondary endpoints were response, progression free and overall survival. The MTD was identified using arithmetic (or fixed) dose escalation. Response rates together with two-sided $95 \%$ confidence intervals were provided for all study endpoints based on categorised responses. Data from all subjects who received any study drug were included in the safety analyses. Overall 
survival (OS) was measured from day 1 of trial entry to death from any cause or last known follow-up; progression-free survival (PFS) from day 1 of trial entry to the first indicator of disease progression, death of any cause, or last known follow-up; Survival curves were estimated using Kaplan-Meier methodology, and log-rank test was used to assess differences between groups. Differences were considered significant if the $p$ value was $<.01$.

\section{Fluorescent in situ hybridisation}

FISH was performed on CD138+ve cells using standard approaches. At least 100 cells were analysed with a cut-off level of $20 \%$. The panel consisted of probes for 1q21/1p36/CEP 1 (Stretton Scientific), CEP12/13q14.3/13q34, IGH break-apart, IGH/FGFR3, IGH/CCND1, IGH/MAF., CEP 5/9/15 (Vysis), p53 (Cytocell Ltd)

\section{RESULTS}

\section{Patient Characteristics:}

A total of 31 patients were enrolled into the study of whom $28(90 \%)$ had previously received thalidomide, 8 (26\%) bortezomib and 20 (65\%) prior autologous stem cell transplant (Table 1). The median age at enrolment was 65 years (range $42-79$ ), median time since the last therapy 7 months (range $1-84$ ) and the median ISS 1 (range $1-3$ ). Five cohorts were enrolled at increasing doses of cyclophosphamide; Cohort 1 (300mg $(n=3))$, cohort 2 (400mg, $(n=6))$, cohort $3(500 m g(n=3))$, cohort $4(600 m g(n=3))$ and cohort 5 (700mg $(n=6))$. Median follow-up at time of analysis is 21 months. 
31 patients $(80 \%)$ had samples taken for cytogenetics and fluorescent in-situ hybridization (FISH) analysis. 10 (33\%) patients failed and 14/31(45\%) were classified as good risk. $7 / 14$ good risk patients had normal cytogenetics. $7 / 31$ (22\%) patients were shown to be in a cytogenetically poor risk category; 4 patients had del13, 2 had t(4:14), 5 had t(14:16) and 1 patient had a del17p.

\section{Toxicity:}

All patients were evaluable for toxicity during cycle 1 (Figure 1, Table 2). Two patients developed grade 4 haematological toxicity in the cohort of patients receiving $700 \mathrm{mg}$ of cyclophosphamide (1 neutropenia and the other pancytopenia) and 3 other patients developed grade 3 neutropenia at a dose of 300, 400 and $600 \mathrm{mg}$. Two further patients developed grade 2 neutropenia at 600 and $700 \mathrm{mg}$ and one other grade 2 neutropenia and anaemia at 400mg. One patient receiving 700mg developed grade 3 syncope, chest pain and pneumonia and was withdrawn from study. Haematological toxicity was therefore established as the dose limiting toxicity (DLT). The maximum tolerated dose of cyclophosphamide in combination with Lenalidomide and dexamethasone was, established at $600 \mathrm{mg}$ daily on days 1 and 8 of a 28 day cycle. A total of 10 more patients were enrolled into the phase 2 part of the study at the $600 \mathrm{mg}$ dose level, and, of these, none experienced a dose limiting toxicity.

During the extended access phase of the study 10 patients $(32 \%)$ developed grade 3 cytopenia in the absence of progressive disease; during cycle 2-5. Two patients in CR developed transient grade 3 neutropenia during cycles 9 and 15 of maintenance both of whom restarted after a 1 week delay. A further 
patient in PR also developed transient grade 3 neutropenia but was able to restart after a delay of 1 week. No non-haematological grade 3 or 4 toxicity was reported. $3(10 \%)$ patients were withdrawn due to metabolic disturbance; 1 patient developed hyperglycaemia during cycle 2 requiring hospital admission and a second developed recurrent hypocalcaemia necessitating withdrawal from study during cycle 10. A third patient developed a raised serum creatinine and hypotension in association with grade 3 thrombocytopenia necessitating a 4 week delay during cycle 3 and was subsequently withdrawn. 2 patients $(6 \%)$ developed a deep vein thrombosis during cycle 5 necessitating a delay in treatment and warfarinisation. Further investigations identified evidence of a pulmonary embolus on CT scan and the other had a low level of possibility on a VQ scan. Two patients (6\%) developed infection; 1 patient died of meningitis during cycle 2 and another patient developed shingles during cycle 16 requiring treatment with acyclovir before restarting lenalidomide maintenance 2 weeks later. 2 patients $(6 \%)$ developed grade 3 cardiac arrhythmias, 1 a bradycardia during cycle 5 necessitating withdrawal from the study and another developed atrial fibrillation during cycle 3 necessitating discontinuation of treatment before being able to restart at the same dose level. No grade 3-4 neurological toxicity was reported but 2 patients (6\%) reported emergent grade 1-2 peripheral neuropathy. Treatment was not discontinued and both patients reported no progression with continuing treatment.

To date, a total of 13 patients have been withdrawn from study, 8 were withdrawn during the induction phase; 3 because of disease progression, 1 with infection; 1 with diabetes mellitus; 1 with an arrhythmia and 2 developed cytopenias. A further 5 patients were withdrawn during the maintenance 
phase; 3 with disease progression, 1 with thrombocytopenia and 1 with hypocalcaemia during cycle 11 . At the beginning of cycle $5,66 \%$ of patients continued on $25 \mathrm{mg}$ daily of Lenalidomide and $64 \%$ of patients completed 9 courses of induction treatment. At the end of the planned 9 cycles of treatment $13(40 \%)$ patients continued on Lenalidomide $25 \mathrm{mg}, 2$ (6.5\%) on $20 \mathrm{mg}$ and 4 (12\%) on $15 \mathrm{mg}$ of daily (Figure 2 ). The most common reason for dose delay and reduction during the first month of treatment was grade 3 neutropenia. There were 9 infectious events reported; 7 grade 1, 1 grade 2, and 1 grade 3 occurring at the maximum dose of $700 \mathrm{mg}$ cyclophosphamide (fig 2). The most commonly observed adverse events were; neutropenia (grade 1-3), cramps (grade 1-2), somnolence (grade 1-2), constipation/diarrhoea (grade 1-2), minor infections (grade 1-2), and musculo-skeletal aches and pains (grade 1).

\section{Determination of MTD:}

During the first 28 days of the study, 2 patients developed a dose limiting toxicity, both of whom were in the $700 \mathrm{mg}$ cyclophosphamide cohort. One had an episode of febrile neutropenia, pneumonia and syncope (all grade 3), and the other grade 4 pancytopenia. The maximum tolerated dose of cyclophosphamide in combination with lenalidomide and dexamethasone was, therefore, established at $600 \mathrm{mg}$ daily on days 1 and 8 of a 28 day cycle.

\section{Clinical Response:}

All patients were evaluable for response. At cycle 1 day 28,4 patients $(13 \%)$ had stable disease (SD), 10 (33\%) had a minimal response (MR), 16 (53\%) had a PR and 1 patient (3\%) achieved a CR. After 9 courses of treatment, the maximum responses seen were CR 29\%, VGPR 7\%, PR 45\%, MR 3\%, SD 
$13 \%$ and PD $3 \%$ (fig 3). By the end of course 6 a maximum response was achieved by 27 patients (90\%), (Figure 3). All patients achieved their maximum response by this time point and no patients had a further improvement in their response following commencement of maintenance therapy. Thus, on an intention to treat basis, $81 \%$ of patients achieved a maximum response of PR or greater and $36 \%>$ VGPR.

Of the ten patients entered into the maximum tolerated dose cohort, 6 achieved PR, and 4 achieved CR. One patient was withdrawn due to progressive disease at cycle 11 of maintenance. There were no deaths and no dose reduction of any study drugs was performed during cycles 1-9.

There was no difference in response rates between those patients with cytogenetic abnormalities and those without $(p=0.73)$ and similarly, the response for those patients with serum beta 2 microglobulin $<2.5$ were not statistically different from those with levels of 2.5 or higher $(p=0.03)$. There were a total of 12 patients who received cyclophosphamide at a dose of less than $600 \mathrm{mg}$ and 19 patients who received a dose of $600 \mathrm{mg}$ or more. No significant differences in response rates were seen between these 2 groups.

After a median follow-up of 21 months, the actuarial median progression free survival (PFS) for the whole group was not reached (Fig 4). At 2 years the PFS is estimated to be $56 \%$. Median actuarial overall survival has also not been reached but was $80 \%$ at 30 months (Fig 5). The 1 patient with deletion of $17 \mathrm{p} 53$ achieved a PR after 2 courses of treatment and has a progression free interval of 17 months. 


\section{DISCUSSION}

This is the first study to establish the maximum tolerated dose of cyclophosphamide in combination with a fixed dose of Lenalidomide and dexamethasone. The combination was well tolerated and the haematological toxicity was not significantly increased despite the advanced age of the patients and heavy pre-treatment, compared to the use of Lenalidomide and dexamethasone alone where $>30 \%$ of patients had only 1 prior treatment and dexamethasone resistance was an exclusion criteria. Importantly, myelotoxicity was easily managed by withholding cyclophosphamide and restarting at a reduced dose. The non-haematological toxicity was low and managed with simple medical intervention that did not require interruption or delay in treatment for the vast majority of subjects. Cardiac arrhythmias is a well known complication of treatment with IMiDs and chemotherapy. 2 of our patients developed grade 3 arrhythmias that necessitated temporary discontinuation of treatment in one and withdrawal from the study in another. In elderly patients receiving this agent it would be prudent therefore to regularly monitor their cardiac status.

The major dose limiting toxicity was haematological with neutropenia developing in $41 \%$ of patients, although grade $3-4$ toxicity was only seen in $19 \%$. Non-haematological grade 3-4 toxicity occurred in less than $5 \%$ of patients with thrombosis occurring in only 2 patients, both of whom restarted treatment after warfarinisation. Higher haematological toxicity has been reported recently with combination of cyclo/ Pred/ Lenalidomide, but at higher doses of cyclophosphamide (Attaya Suvannasankha 2009, Donna E. Reece 2009). Importantly, toxicity figures in our study are similar to those 
seen in MM009 and MM010 studies, suggesting that cyclophosphamide does not add significantly to haematological toxicity. The toxicity profile also seems to differ from the combination of melphalan and prednisolone with Lenalidomide where Grade 3-4 haematological toxicity at a level of $52.4 \%$ was seen at the maximum tolerated dose level (Palumbo, et al 2007). The difference in myelosuppression with cyclophosphamide compared to melphalan is consistent with the CRD combination being a safer and more tolerable regimen than MPR.

The addition of cyclophosphamide in this study has increased the overall response rate $[C R+V G P R+P R]$ to $81 \%$ which is superior to that reported for Lenalidomide alone (17\%-23\%) (Armoiry, et al 2008) or in combination with dexamethasone (48\%-51\%) (Wang, et al 2008). These responses were observed despite the number of median lines of prior therapy of 3 (range 1-6), used in this study compared to that seen in the MM009 and MM010 trials where up to one third of patients were treated at first relapse. These responses are also higher than our previously reported $C R+P R$ response rates of $65 \%$ in a retrospective audit of patients receiving a combination of Lenalidomide, cyclophosphamide and dexamethasone (Morgan, et al 2007), all of whom had end-stage disease.

Responses after relapse from first line treatment for myeloma are generally short-lived, with outcomes being determined by the presence of adverse comorbidities, cytogenetic abnormalities and the adverse side-effects profile of re-induction treatment. In both first and subsequent relapses, increasing drug resistance and accumulated myelo- and nephrotoxicity restrict the intensity of 
drug that can safely be delivered. There are few studies examining the survival of patients after first relapse. A retrospective analysis of 2528 patients entered into the non-intensive MRC Myeloma IV, V, VI and VII trials between 1980 and 1997, who achieved a stable plateau phase (Augustson, et al 2005) showed that the median OS of the 1372 patients who achieved plateau was 3.9 years. At relapse, for those with 1 prior therapy it was 16.1 months and 9.2 for $\geq 2$ prior therapies. If duration of response is taken into account in those whose duration of plateau phase was $<1 \mathrm{yr}, 1-3 \mathrm{yrs}$ and $>3 \mathrm{yrs}$, the median survivals from relapse were 11,17 and 21 months, respectively. The mean duration of survival at first, second and third plateau were $4.8,2.0$ and 1.0 years respectively. In a more recent study of patients with relapsed/refractory disease(Quach, et al 2009), progression free survival beyond 2 years was seen in only $27 / 141$ (19\%). The predicted two year PFS of $56 \%$ within the CRD combination would support the hypothesis that Revlimid adds significantly to the effects of cyclophosphamide and dexamethasone alone and represents a significant step forward.

It has been suggested (Stewart, et al 2007) that the presence of $t(4 ; 14)$, $t(14 ; 16), t(14 ; 20)$ and del17p13 on FISH and del 13 on metaphase cytogenetics are associated with high risk disease. Although the numbers are too small to draw firm conclusions, a quarter of patients in this study were classified as high risk, but there was no difference seen in response to treatment compared to standard risk patients (93\% versus $85 \%$; data not shown). Similar results have been reported by the Canadian group (Reece, et al 2009). Our results support these findings they need to be confirmed in 


\section{a larger study before the CRD regimen can be recommended as a good therapeutic option for patients with poor cytogenetic features.}

The responses seen with the CRD regimen compared well with the results of other phase I/II studies of Lenalidomide combinations. The combination of Lenalidomide, dexamethasone with liposomal doxorubicin and vincristine, was associated with a response rate of $75 \%$ and $\mathrm{CR} / \mathrm{nCR}$ rate of $29 \%$ but the maximum tolerated dose of Lenalidomide was only $10 \mathrm{mg} / \mathrm{day}(\mathrm{Baz}$, et al 2006). More recently, a Phase I/II study by the German Myeloma Study Group, DSMM(Knop, et al 2009), using Lenalidomide in combination with dexamethasone, conventional doxorubicin and pegfilgrastim support, was associated with an ORR of $69 \%(\mathrm{CR}+\mathrm{VGPR}+\mathrm{PR})$. They further reported, at a median follow-up of 14.6 months a median time to disease progression of 45 weeks and the one year survival probability was $88 \%$. In our study, the overall survival of $80 \%$ at 30 months is encouraging. The German Group further report a higher percentage of grade $3 / 4$ neutropenia (48\%) and thrombocytopenia (38\%) compared to what we observed here in combination with cyclophosphamide. The thromboembolic complication rate we report is similar to the DSMM study, but grade 3/4 infections were less common ( $3 \%$ vs $10 \%)$

The optimal combination of novel agents and chemotherapy drugs will vary between individual patients according to their prior responses, suitability for stem cell transplant and co-morbidities. The response rate in this study is high and the PFS and OS are impressive and they need to be substantiated in a larger phase II study. As new agents become available we need to identify 
how best to use them in combination to optimise response rate and duration of responses by conducting well designed clinical trials. At relapse the combination of Lenalidomide with cyclophosphamide and dexamethasone appears to be well tolerated with encouraging responses and outcomes and these findings should be confirmed in larger randomised trials in the future.

Acknowledgements: The authors acknowledge financial support from the Department of Health via the National Institute for Health Research (NIHR) comprehensive Biomedical Research Centre award to Guy's \& St Thomas' NHS Foundation Trust in partnership with King's College London and King's College Hospital NHS Foundation Trust. Cancer Research UK and the Celgene Corporation also provided financial support for the trial administration

\section{Authorship contributions:}

Designed research: SS, GM, FD and KP

Performed research: SS, GM, FD, KR, MJ and KB

Collected data, analyzed and interpreted data: SC, BH and DL

Wrote the manuscript: SS, GM, FD and KR

No conflicts of interest to disclose 


\section{REFERENCES}

Armoiry, X., Aulagner, G. \& Facon, T. (2008) Lenalidomide in the treatment of multiple myeloma: a review. J Clin Pharm Ther, 33, 219-226.

Attaya Suvannasankha, S.F., Robin A O'Bryant, Lisa L Wood, and Rafat Abonour (2009) Phase 2 Clinical Trial of Oral Lenalidomide, Cyclophosphamide, and Prednisone (RCP) for Newly Diagnosed Multiple Myeloma Patients. Blood, 114, 2882.

Augustson, B.M., Begum, G., Dunn, J.A., Barth, N.J., Davies, F., Morgan, G., Behrens, J., Smith, A., Child, J.A. \& Drayson, M.T. (2005) Early mortality after diagnosis of multiple myeloma: analysis of patients entered onto the United kingdom Medical Research Council trials between 1980 and 2002--Medical Research Council Adult Leukaemia Working Party. J Clin Oncol, 23, 9219-9226.

Baz, R., Walker, E., Karam, M.A., Choueiri, T.K., Jawde, R.A., Bruening, K., Reed, J., Faiman, B., Ellis, Y., Brand, C., Srkalovic, G., Andresen, S., Knight, R., Zeldis, J. \& Hussein, M.A. (2006) Lenalidomide and pegylated liposomal doxorubicin-based chemotherapy for relapsed or refractory multiple myeloma: safety and efficacy. Ann Oncol, 17, 1766-1771.

Berenson, J.R., Yang, H.H., Sadler, K., Jarutirasarn, S.G., Vescio, R.A., Mapes, R., Purner, M., Lee, S.P., Wilson, J., Morrison, B., Adams, J., Schenkein, D. \& Swift, R. (2006) Phase I/II trial assessing bortezomib and melphalan combination therapy for the treatment of patients with relapsed or refractory multiple myeloma. J Clin Oncol, 24, 937-944.

Child, J.A., Morgan, G.J., Davies, F.E., Owen, R.G., Bell, S.E., Hawkins, K., Brown, J., Drayson, M.T. \& Selby, P.J. (2003) High-dose chemotherapy with hematopoietic stem-cell rescue for multiple myeloma. $N$ Engl J Med, 348, 1875-1883.

Dimopoulos, M., Spencer, A., Attal, M., Prince, H.M., Harousseau, J.L., Dmoszynska, A., San Miguel, J., Hellmann, A., Facon, T., Foa, R., Corso, A., Masliak, Z., Olesnyckyj, M., Yu, Z., Patin, J., Zeldis, J.B. \& Knight, R.D. (2007) Lenalidomide plus dexamethasone for relapsed or refractory multiple myeloma. $N$ Engl J Med, 357, 2123-2132.

Donna E. Reece, E.M.-K., Arooj Khan, Saima Dean, Peter Anglin, Christine Chen, Vishal Kukreti, Joseph R. Mikhael, and Suzanne Trudel (2009) Phase I-II Trial of Oral Cyclophosphamide, Prednisone and Lenalidomide (Revlimid®) (CPR) for the Treatment of Patients with Relapsed and Refractory Multiple Myeloma. Blood, 114.

Durie, B.G., Harousseau, J.L., Miguel, J.S., Blade, J., Barlogie, B., Anderson, K., Gertz, M., Dimopoulos, M., Westin, J., Sonneveld, P., Ludwig, H., Gahrton, G., Beksac, M., Crowley, J., Belch, A., Boccadaro, M., Cavo, M., Turesson, I., Joshua, D., Vesole, D., Kyle, R., Alexanian, R., Tricot, G., Attal, M., Merlini, G., Powles, R., Richardson, P., Shimizu, K., Tosi, P., Morgan, G. \& Rajkumar, S.V. (2006) International uniform response criteria for multiple myeloma. Leukemia, 20, 14671473.

Knop, S., Gerecke, C., Liebisch, P., Topp, M.S., Platzbecker, U., Sezer, O., Vollmuth, C., Falk, K., Glasmacher, A., Maeder, U., Einsele, H. \& Bargou, R.C. (2009) Lenalidomide $(\operatorname{Revlimid}(\mathrm{R}))$, adriamycin and dexamethasone (RAD) in patients with relapsed and refractory multiple myeloma: A report from the German Myeloma Study Group DSMM (Deutsche Studiengruppe Multiples Myelom). Blood.

Kropff, M., Bisping, G., Schuck, E., Liebisch, P., Lang, N., Hentrich, M., Dechow, T., Kroger, N., Salwender, H., Metzner, B., Sezer, O., Engelhardt, M., Wolf, H.H., Einsele, H., Volpert, S., Heinecke, A., Berdel, W.E. \& Kienast, J. (2007) Bortezomib 
in combination with intermediate-dose dexamethasone and continuous low-dose oral cyclophosphamide for relapsed multiple myeloma. Br J Haematol, 138, 330-337.

Morgan, G.J., Schey, S.A., Wu, P., Srikanth, M., Phekoo, K.J., Jenner, M. \& Davies, F.E. (2007) Lenalidomide (Revlimid), in combination with cyclophosphamide and dexamethasone (RCD), is an effective and tolerated regimen for myeloma patients. $\mathrm{Br}$ J Haematol, 137, 268-269.

Palumbo, A., Bringhen, S., Caravita, T., Merla, E., Capparella, V., Callea, V., Cangialosi, C., Grasso, M., Rossini, F., Galli, M., Catalano, L., Zamagni, E., Petrucci, M.T., De Stefano, V., Ceccarelli, M., Ambrosini, M.T., Avonto, I., Falco, P., Ciccone, G., Liberati, A.M., Musto, P. \& Boccadoro, M. (2006) Oral melphalan and prednisone chemotherapy plus thalidomide compared with melphalan and prednisone alone in elderly patients with multiple myeloma: randomised controlled trial. Lancet, 367, 825831.

Palumbo, A., Falco, P., Corradini, P., Falcone, A., Di Raimondo, F., Giuliani, N., Crippa, C., Ciccone, G., Omede, P., Ambrosini, M.T., Gay, F., Bringhen, S., Musto, P., Foa, R., Knight, R., Zeldis, J.B., Boccadoro, M. \& Petrucci, M.T. (2007) Melphalan, prednisone, and lenalidomide treatment for newly diagnosed myeloma: a report from the GIMEMA--Italian Multiple Myeloma Network. J Clin Oncol, 25, 4459-4465.

Quach, H., Mileshkin, L., Seymour, J.F., Milner, A., Ritchie, D., Harrison, S., Westerman, D. \& Prince, H.M. (2009) Predicting durable remissions following thalidomide therapy for relapsed myeloma. Leuk Lymphoma, 50, 223-229.

Raje, N., Powles, R., Kulkarni, S., Milan, S., Middleton, G., Singhal, S., Mehta, J., Millar, B., Viner, C., Raymond, J., Treleaven, J., Cunningham, D. \& Gore, M. (1997) A comparison of vincristine and doxorubicin infusional chemotherapy with methylprednisolone (VAMP) with the addition of weekly cyclophosphamide (CVAMP) as induction treatment followed by autografting in previously untreated myeloma. Br J Haematol, 97, 153-160.

Reece, D., Song, K.W., Fu, T., Roland, B., Chang, H., Horsman, D.E., Mansoor, A., Chen, C., Masih-Khan, E., Trieu, Y., Bruyere, H., Stewart, D.A. \& Bahlis, N.J. (2009) Influence of cytogenetics in patients with relapsed or refractory multiple myeloma treated with lenalidomide plus dexamethasone: adverse effect of deletion $17 \mathrm{p} 13$. Blood.

Sidra, G., Williams, C.D., Russell, N.H., Zaman, S., Myers, B. \& Byrne, J.L. (2006) Combination chemotherapy with cyclophosphamide, thalidomide and dexamethasone for patients with refractory, newly diagnosed or relapsed myeloma. Haematologica, 91, 862-863.

Singhal, S., Mehta, J., Desikan, R., Ayers, D., Roberson, P., Eddlemon, P., Munshi, N., Anaissie, E., Wilson, C., Dhodapkar, M., Zeddis, J. \& Barlogie, B. (1999) Antitumor activity of thalidomide in refractory multiple myeloma. $N$ Engl J Med, 341, $1565-1571$.

Stewart, A.K., Bergsagel, P.L., Greipp, P.R., Dispenzieri, A., Gertz, M.A., Hayman, S.R., Kumar, S., Lacy, M.Q., Lust, J.A., Russell, S.J., Witzig, T.E., Zeldenrust, S.R., Dingli, D., Reeder, C.B., Roy, V., Kyle, R.A., Rajkumar, S.V. \& Fonseca, R. (2007) A practical guide to defining high-risk myeloma for clinical trials, patient counseling and choice of therapy. Leukemia, 21, 529-534.

Wang, M., Dimopoulos, M.A., Chen, C., Cibeira, M.T., Attal, M., Spencer, A., Rajkumar, S.V., Yu, Z., Olesnyckyj, M., Zeldis, J.B., Knight, R.D. \& Weber, D.M. (2008) Lenalidomide plus dexamethasone is more effective than dexamethasone alone in patients with relapsed or refractory multiple myeloma regardless of prior thalidomide exposure. Blood, 112, 4445-4451. 
Weber, D.M., Chen, C., Niesvizky, R., Wang, M., Belch, A., Stadtmauer, E.A., Siegel, D., Borrello, I., Rajkumar, S.V., Chanan-Khan, A.A., Lonial, S., Yu, Z., Patin, J., Olesnyckyj, M., Zeldis, J.B. \& Knight, R.D. (2007) Lenalidomide plus dexamethasone for relapsed multiple myeloma in North America. $N$ Engl J Med, 357, 2133-2142.

Yakoub-Agha, I., Attal, M., Dumontet, C., Delannoy, V., Moreau, P., Berthou, C., Lamy, T., Grosbois, B., Dauriac, C., Dorvaux, V., Bay, J.O., Monconduit, M., Harousseau, J.L., Duguet, C., Duhamel, A. \& Facon, T. (2002) Thalidomide in patients with advanced multiple myeloma: a study of 83 patients--report of the Intergroupe Francophone du Myelome (IFM). Hematol J, 3, 185-192.

\section{Table 1 Patient Characteristics}

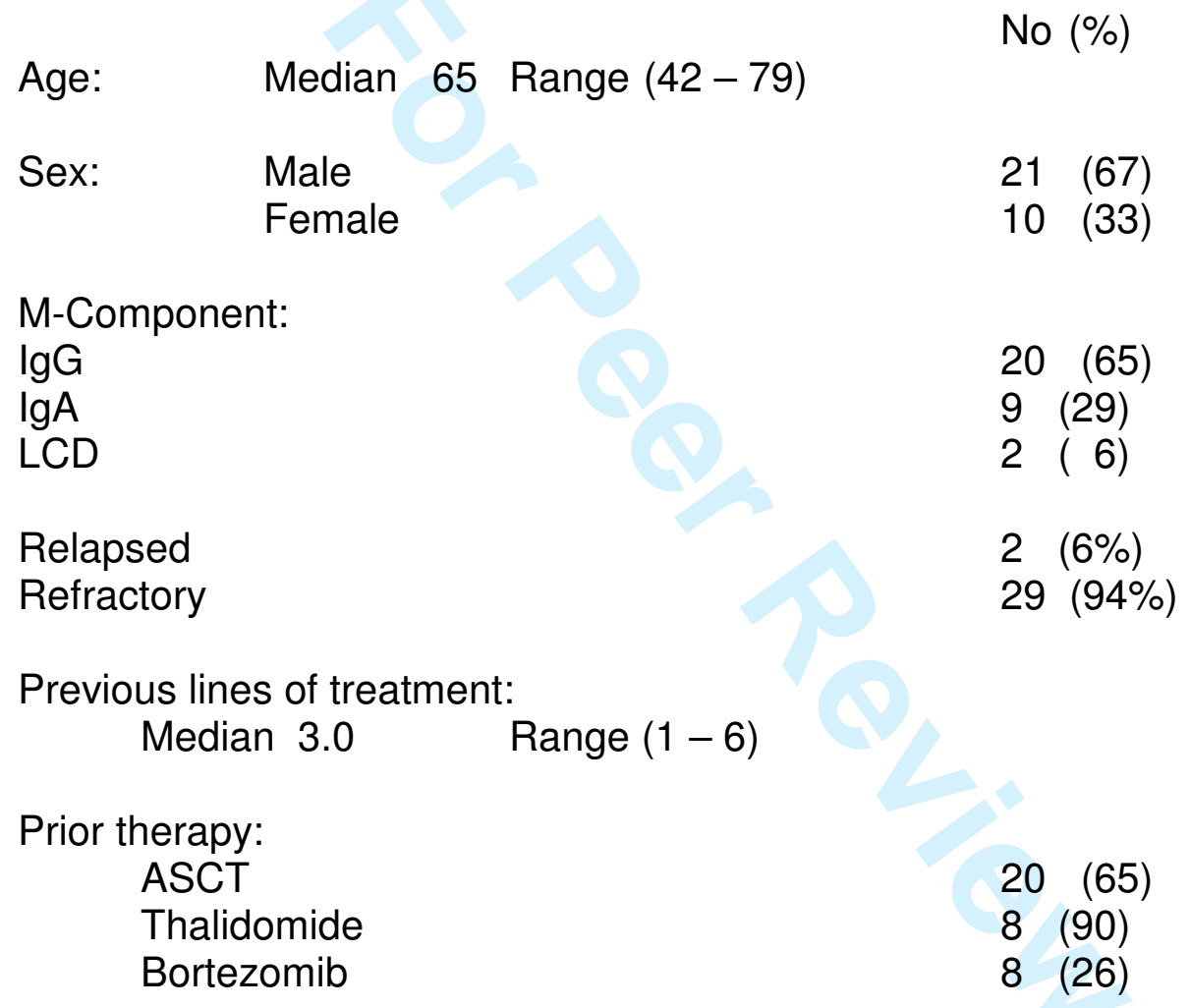

Serum albumin:

Median 39 Range (21-45)

Serum $\beta_{2} m$ :

Median $\quad 2.5$ Range $(1.1-12.9)$

Adverse FISH

n $(\%)$

Total assessable $\quad 21(67)$

del $13 q \quad 4 \quad(13)$

$\mathrm{t}(4: 14) \quad 2 \quad(6)$

$\mathrm{t}(14: 16) \quad 5 \quad(16)$

del $17 \mathrm{p} 53$ 
Table 2. Haematological Toxicity. The number of adverse haematological events (all grades) occurring at each cyclophosphamide dose level.

\begin{tabular}{lcccc}
\multicolumn{4}{c}{ Haematological Toxicity } \\
No of Patients & \multicolumn{4}{c}{ Number of patients } \\
\cline { 2 - 5 } Dose(mgs) & G1 & G2 & G3 & G4 \\
\hline 300 & 0 & 0 & 1 & 0 \\
400 & 1 & 1 & 1 & 0 \\
500 & 0 & 0 & 0 & 0 \\
600 & 0 & 1 & 1 & 0 \\
700 & 0 & 1 & 0 & 2 \\
Total & 1 & 3 & 3 & 2 \\
\hline
\end{tabular}


Figure 1. Non Haematological Toxicity. Frequency of nonhaematological toxicity during the initial 4 weeks of treatment (all grades)

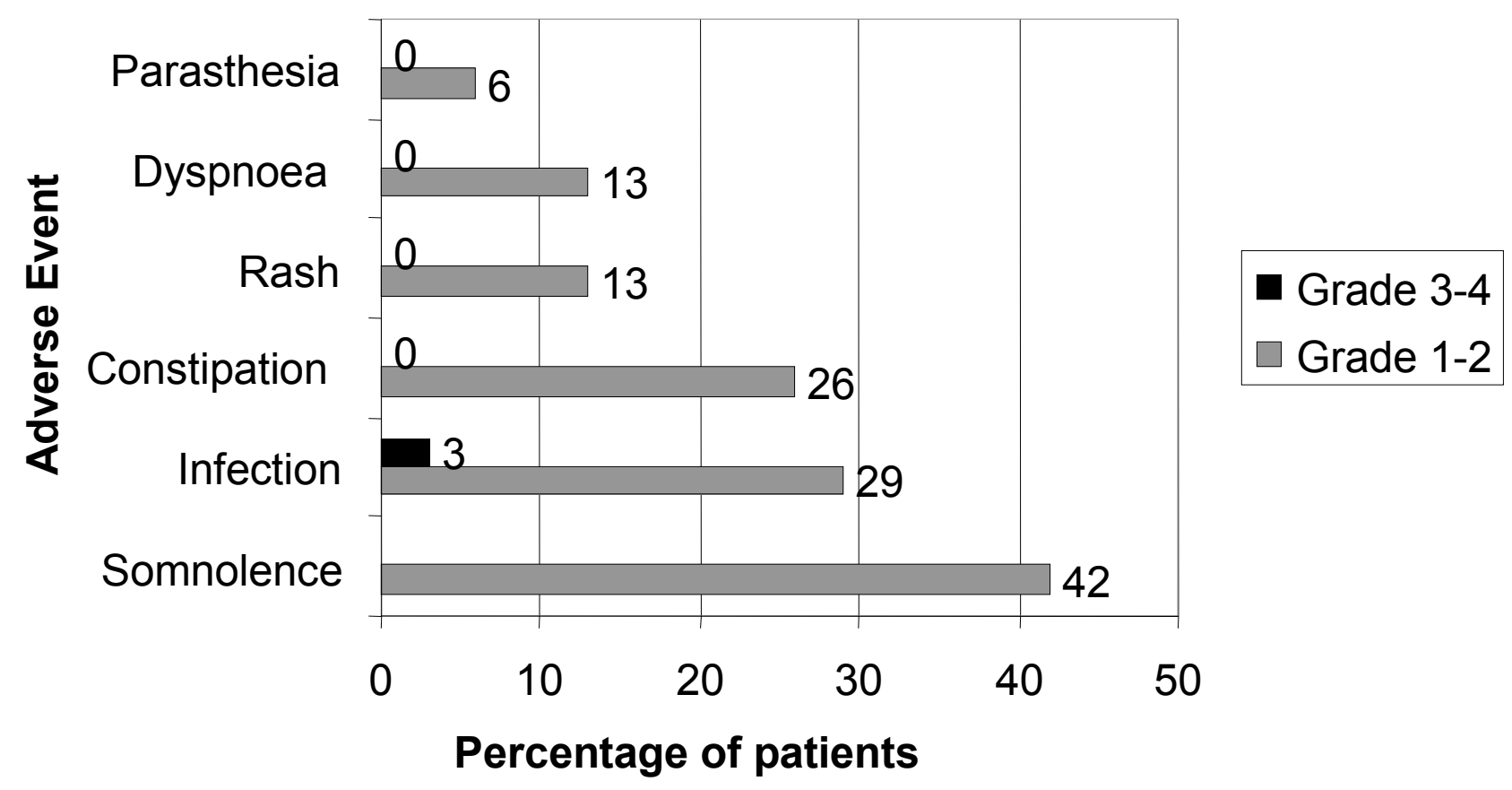


Figure 2: Dose modification throughout the induction phase of the study course 1 through 9

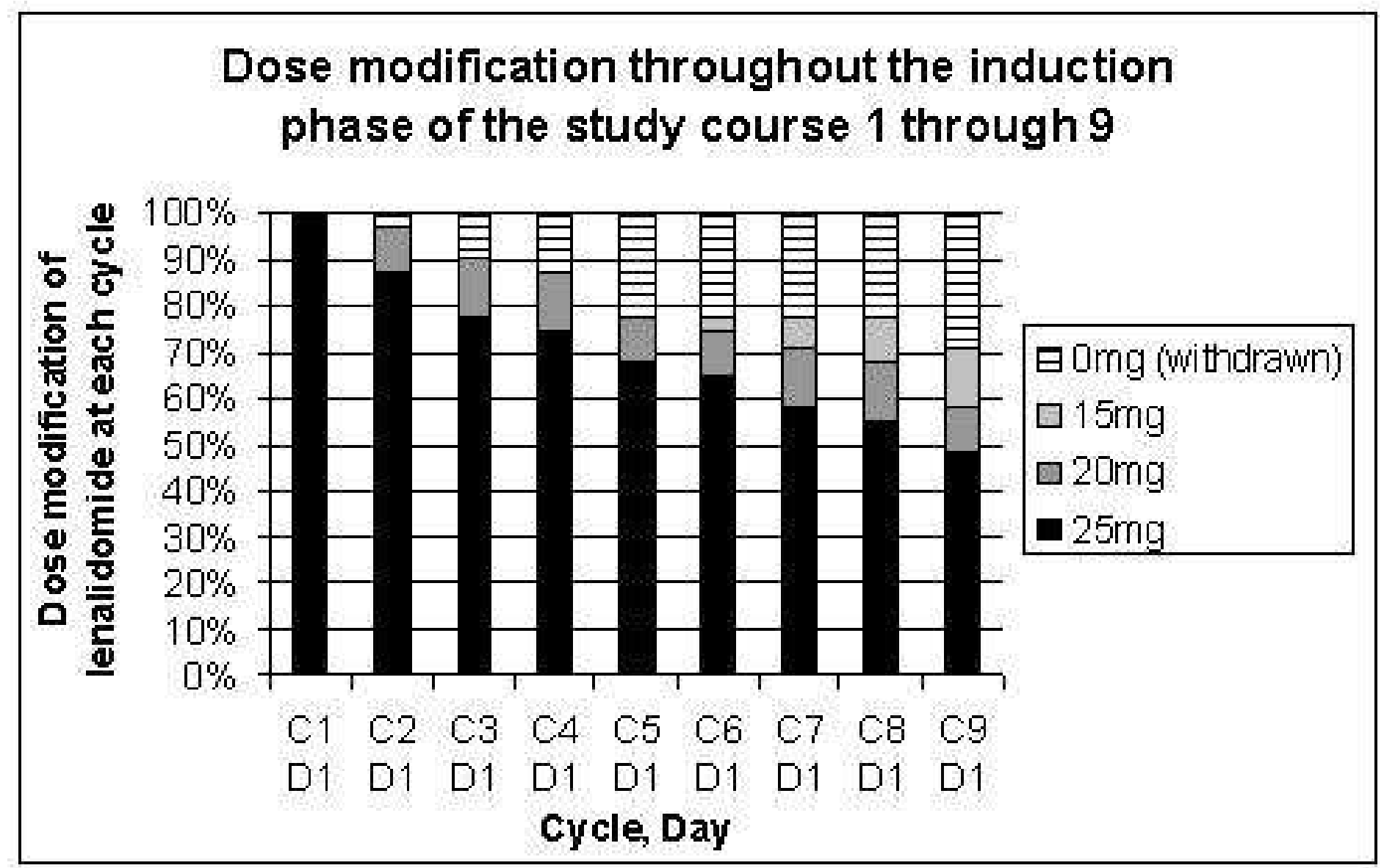

$\mathrm{C} 1$ is course 1 and D1 is day 1 of treatment cycle 
Figure 3: Overall response rate. Percentage of patients achieving maximum response to treatment.

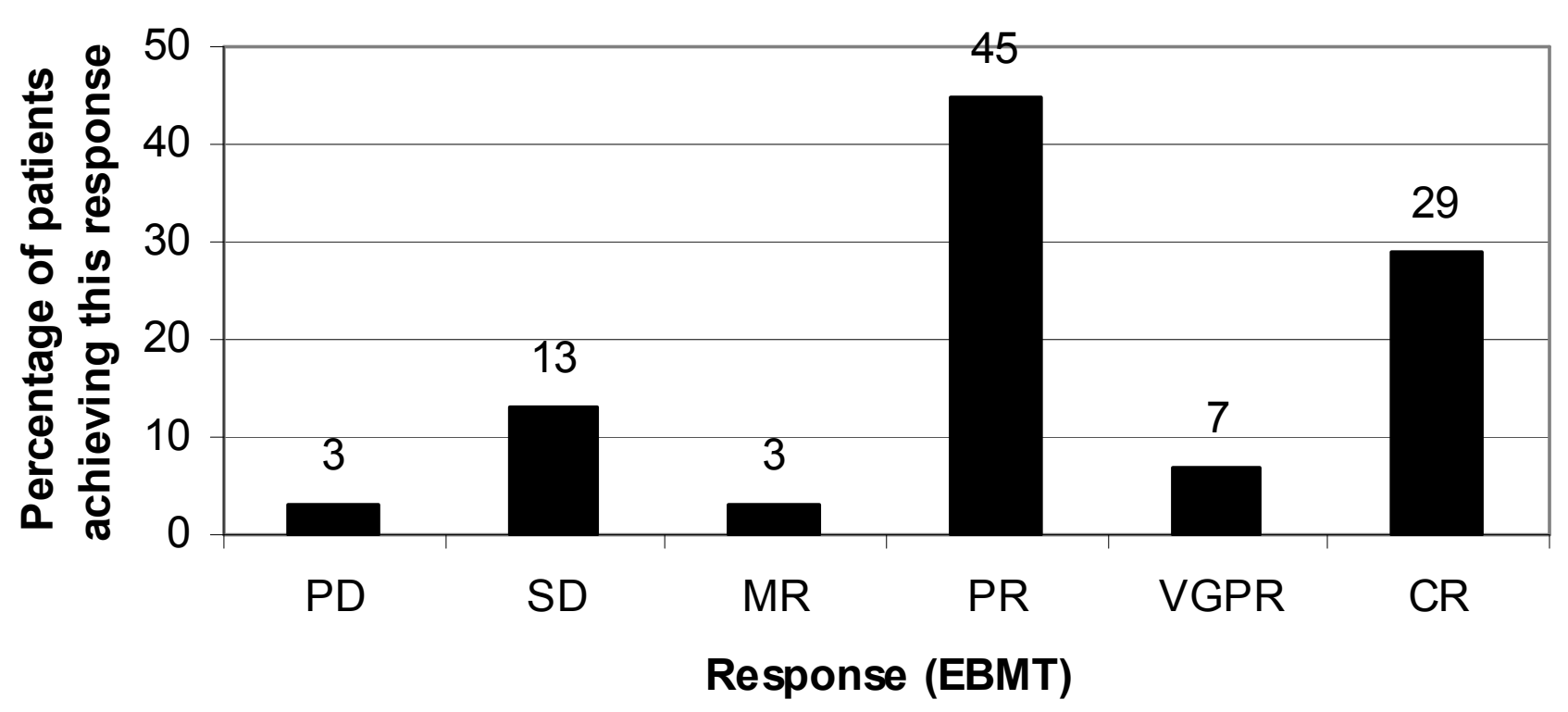




\section{Figure 4}
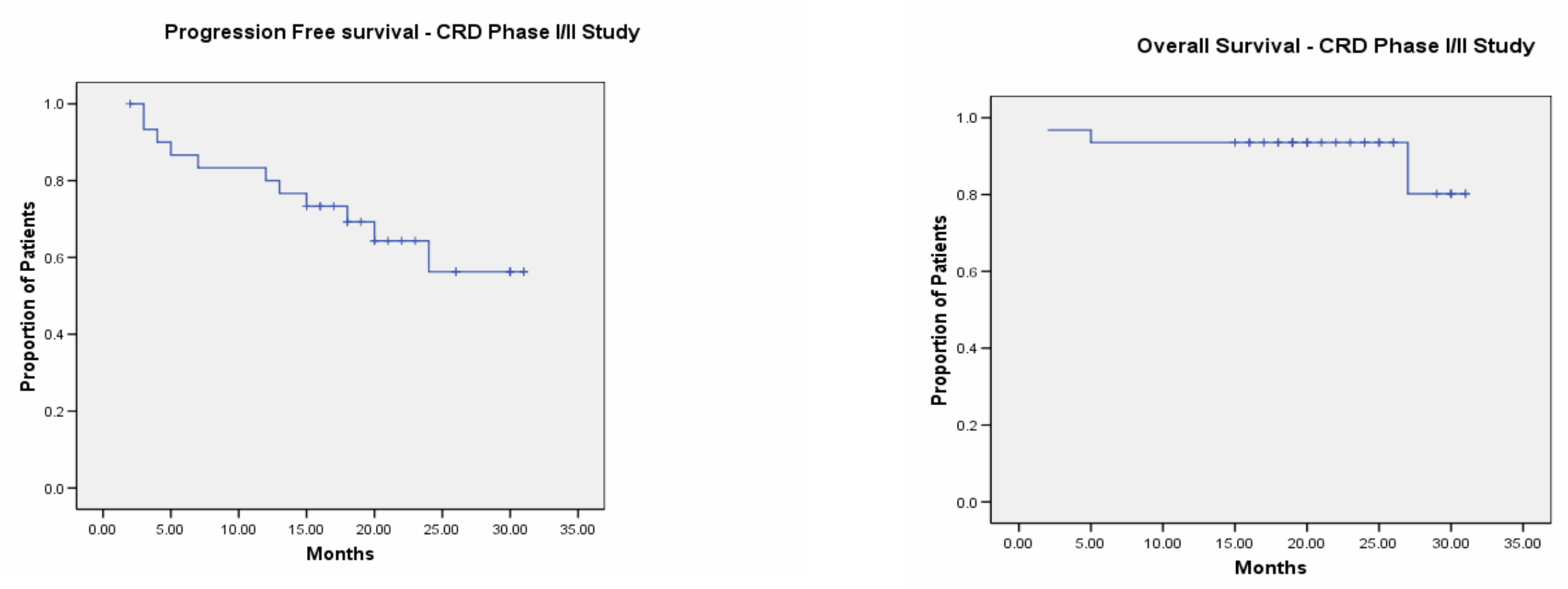